\title{
Emociones académicas
}

\section{y aprendizaje de biología, una asociación duradera}

\section{Academic emotions and the learning of biology, a long-lasting association}

\author{
Jesús A. G. Ochoa de Alda, José María Marcos-Merino \\ Departamento de Didáctica de las Ciencias Experimentales y las Matemáticas, Universidad de Extremadura \\ ochoadealda@unex.es,jmmarcos@unex.es \\ Francisco Javier Méndez Gómez \\ Departamento de Psicología y Antropología, Universidad de Extremadura, Cáceres, España \\ fimendezgomez@gmail.com \\ Vicente Mellado Jiménez, M. Rocío Esteban Gallego \\ Departamento de Didáctica de las Ciencias Experimentales y las Matemáticas, Universidad de Extremadura, \\ vmellado@unex.es, rocioesteban@unex.es
}

RESUMEN • Los alumnos experimentan una gran diversidad de emociones en el ámbito académico. Estas emociones y el aprendizaje se condicionan recíprocamente, lo que sugiere que el resultado del aprendizaje que perdura desde etapas educativas previas podría estar relacionado con las emociones experimentadas entonces. Para comprobarlo, en este trabajo se comparan los resultados de aprendizaje de Biología que perduran desde la Educación Secundaria con el recuerdo de algunas emociones experimentadas durante dicha etapa, en dos contextos educativos, en una muestra de 152 futuros maestros. Los resultados indican que existe una asociación significativa entre ese recuerdo y el aprendizaje de conceptos biológicos adquiridos en Secundaria.

PALABRAS CLAVE: Emociones académicas; Aprendizaje duradero; Cuestionario autoinforme; Biología.

ABSTRACT - The classroom is an emotional place. There is virtually no major human emotion which is not experienced in academic settings. Emotional state and learning influence each other, suggesting that a long-lasting association may be maintained. To assess this possibility, this contribution explores the association between biology learning outcomes, perpetuated from Secondary Education, and past emotions experienced at this stage (in two different academic settings) by a sample of 152 pre-service teachers. Correlation analysis supports a significant relationship between past emotions and longlasting learning outcomes of key Biology concepts acquired during Secondary education.

KEYWORDS: Academic emotions; Long-lasting learning; Self-report test; Biology.

Recepción: febrero 2018 • Aceptación: septiembre 2018 • Publicación: junio 2019 


\section{INTRODUCCIÓN}

La adaptabilidad es una característica esencial para la supervivencia de los seres vivos. El proceso adaptativo implica la percepción e integración de las señales internas y externas, así como la posterior elaboración de una respuesta apropiada. En los seres vivos, tanto la percepción como la integración de las señales están moduladas por su estado interno, que realiza una evaluación subjetiva para determinar si estas son «buenas» o «malas» para el individuo y que, a su vez, controla distintas acciones fisiológicas con las que responder. En los humanos, ese estado interno está gobernado por las emociones, que activan una serie de cambios cognitivos, fisiológicos y motores, como resultado de la determinación de que un estímulo interno (hambre, dolor, expectativas, recuerdos...) o externo (ruido, dificultad...) es beneficioso o perjudicial. A pesar de que no se ha establecido una definición única de emoción, existe consenso respecto a que las emociones reflejan la capacidad de valorar acontecimientos en un determinado contexto y estimar si son deseables para el individuo (Damasio, 2010).

Para describir las emociones se emplean habitualmente varias dimensiones como la valencia (grado en que una emoción es beneficiosa, positiva; o perjudicial, negativa), la excitación o activación (grado en que una emoción provoca alerta sensorial, movilidad y disposición para responder) y el grado de acercamiento o elusión que provoca respecto a una determinada acción (Mauss y Robinson, 2009). Estas dimensiones han sido corroboradas por estudios neurofisiológicos que han confirmado que existen pautas de activación neuronal correspondientes a las diferentes dimensiones comunes entre individuos (Kassam et al., 2013). La complejidad de los procesos emocionales y la diversidad de las respuestas fisiológicas asociadas dificultan la posibilidad de integrar las emociones en una medida discreta, más aún si se considera que las emociones son procesos muy variables, dado su carácter individual, situacional e integrador de experiencias pasadas. Uno de los métodos más utilizados para medir emociones es el muestreo mediante cuestionarios en los que los participantes autoinforman de sus emociones. Este método es fácil de implementar y proporciona medidas cuya varianza (entre el 50 y $60 \%$ ) puede ser explicada por las dimensiones de valencia y excitación. Sin embargo, se ha descrito que la medida de las emociones, obtenida mediante autoinformes, sufre algunos sesgos relacionados con la dificultad de los individuos de recordar emociones pasadas y diferenciarlas de las emociones presentes hacia una determinada situación similar (Robinson y Clore, 2002).

\section{Las emociones en el aula condicionan el aprendizaje}

Las aulas son ambientes emocionales: en el ámbito académico se experimentan las principales emociones humanas como ansiedad, sorpresa, alegría, aburrimiento... Estas emociones influyen en los procesos de aprendizaje. La sorpresa, por ejemplo, permite fijar la atención sobre algo que de otra manera hubiese pasado inadvertido, mientras que el aburrimiento contribuye a la pérdida progresiva de atención. Aunque en general las emociones positivas (como la alegría) se asocian positivamente con el aprendizaje, y las negativas (como el aburrimiento) se asocian negativamente, esta asociación puede ser tanto directa (por ejemplo, un poco de nerviosismo puede mejorar el aprendizaje) como inversa (mucho nerviosismo inhibe el aprendizaje) (Tyng et al., 2017). Además, esta asociación depende tanto de la asignatura como del contexto académico (Goetz et al., 2007; Pekrun et al., 2011). Estudios neurofisiológicos muestran como, en el cerebro, las emociones y diferentes procesos cognitivos (memoria, atención o capacidad de resolver problemas) están integrados en áreas críticas para la regulación del flujo de información entre diferentes regiones ( $\mathrm{Gu}$ et al., 2013). De modo que las emociones académicas pueden tener un efecto importante sobre el aprendizaje a través del control de la atención, de la motivación para aprender y sobre la elección de las estrategias de aprendizaje (Mega, Ronconi y De Beni, 2014). 
La información relacionada con algún estímulo emocional se recuerda mejor que la neutra (Dunsmoor et al., 2015) ya que las emociones facilitan la codificación, o creación de la memoria sobre un determinado evento, y ayudan a recuperar esta información de manera eficiente en el largo plazo (Tyng et al., 2017). Así, se ha comprobado que una activación de las emociones positivas puede dar lugar a mejores resultados de aprendizaje (Kensinger y Corkin, 2004).

\section{El aprendizaje condiciona las emociones}

Estudios neurofisiológicos han mostrado que el estado emocional y el aprendizaje están recíprocamente condicionados: el domino afectivo configura al cognitivo y viceversa (Eldar y Niv, 2015). El estado emocional tiene un efecto sobre la estimación anticipada de la recompensa asociada a una acción (p. ej. estudiar), mientras que la precisión con la que se ha hecho esta estimación, una vez obtenida dicha recompensa (p. ej. la nota), modifica a su vez el estado emocional (Eldar et al., 2016). Estas observaciones apoyan la teoría psicológica del Control-Valor (Pekrun, 2006), que explica cómo las emociones de los individuos, en relación con las actividades de enseñanza-aprendizaje, están moduladas por el control sobre esas actividades y por el valor esperado. Así, las emociones experimentadas durante el desarrollo de las actividades y tras los resultados de aprendizaje influyen tanto en la estimación del valor de futuros procesos de enseñanza-aprendizaje como en sus resultados (Pekrun et al., 2014; Putwain et al., 2017). Algunos estudios apoyan que, en el caso de las Matemáticas (asignatura relacionada con emociones como nerviosismo o ansiedad), su aprendizaje duradero podría estar mediado por las emociones experimentadas durante su adquisición (Murayama et al., 2013; Pekrun et al. 2017). Sin embargo, en el caso de la Biología, relacionada con emociones positivas (Mellado et al., 2014), esta circunstancia no ha sido aún explorada.

\section{Las emociones en la labor docente}

Dada la influencia de las emociones en el aprendizaje, resulta fundamental tenerlas en cuenta durante su labor docente. La investigación actual aconseja que en el aula (Pekrun, 2014): a) se considere el carácter individual de las emociones; $b$ ) se promueva el disfrute durante el aprendizaje; $c$ ) se eviten las emociones negativas en exceso y, si surgiesen, se ayudase al alumno a emplearlas de forma productiva; d) se promueva la autoestima y el interés por las tareas académicas; $e$ ) se ayude a los estudiantes a regular sus emociones, y $f$ ) se utilicen las emociones positivas del profesor para promover estas entre los alumnos. En relación con esta última recomendación, cabe destacar, como ejemplo, que el disfrute experimentado por un grupo de 71 profesores mientras impartían Matemáticas se correlacionó con el experimentado por sus 1.763 alumnos mientras aprendían esta materia (Frenzel et al., 2009). Por tanto, las emociones experimentadas por los docentes hacia su materia y la enseñanza de esta se transfieren a sus alumnos. Debido a esta capacidad moduladora de emociones del docente, resulta necesario abordar la interacción entre las emociones y el aprendizaje desde la formación inicial del profesorado.

\section{Las emociones en el aprendizaje de las ciencias de los maestros en formación}

Los maestros en formación muestran un gran número de emociones académicas en relación con diferentes tipos de actividades de ciencias (prácticas, exposiciones, debates...). En estos estudiantes, se ha observado que las emociones positivas que experimentaron en las clases de ciencias de Educación Primaria fueron menos frecuentes durante la Educación Secundaria. En esta etapa destacaban emociones como miedo o agobio, al tiempo que atribuían poco valor a la ciencia, que era descrita como una disciplina aburrida, difícil e irrelevante (Mellado et al., 2014). Esta asociación entre algunas emociones académicas (presentes y pasadas) y el valor que se atribuye a las ciencias y su aprendizaje concuerda 
con lo esperado según la teoría del Control-Valor de Pekrun. Se podría argumentar que el tiempo minimiza el impacto de las emociones sobre el aprendizaje y que este impacto, a largo plazo, es irrelevante. Sin embargo, en una muestra de maestros en formación de varias universidades españolas se ha observado, de manera consistente, una relación entre sus emociones pasadas (en Educación Primaria y Secundaria) hacia las ciencias, sus emociones presentes hacia las ciencias y las emociones con las que creen que van a transmitir las ciencias como docentes (Mellado et al., 2014). De manera que si, como se ha indicado anteriormente, las emociones determinan la manera de aprender y enseñar, las emociones experimentadas por los maestros en formación afectarán a su motivación y a su forma de enseñar a las generaciones futuras. Por ello es necesario comprender la influencia de las diferentes actividades formativas en las emociones y el aprendizaje de los futuros maestros.

Actualmente, desde nuestro grupo de investigación, estamos indagando en el papel de las emociones en el aprendizaje de las ciencias de maestros en formación mediante actividades prácticas. Resultados preliminares muestran que, a corto plazo, existe una asociación significativa entre el aprendizaje de Biología adquirido mediante prácticas y las emociones positivas experimentadas durante estas (Marcos-Merino, Esteban y Ochoa de Alda, 2016), si bien se desconoce si esta asociación se mantiene a largo plazo.

\section{PROBLEMA DE INVESTIGACIÓN}

El objetivo principal es determinar si existe una asociación significativa entre el recuerdo de las emociones, experimentadas en Educación Secundaria, y el aprendizaje de Biología adquirido y retenido desde esa etapa educativa, en una muestra de estudiantes del Grado en Educación Primaria de la Universidad de Extremadura. Para ello se plantean los siguientes objetivos específicos:

- Determinar la intensidad de diez emociones, tanto durante las clases expositivas y las clases prácticas de ciencias de Educación Secundaria (emociones pasadas), como antes de la realización de una práctica de ciencias (emociones presentes).

- Establecer si la muestra diferencia la intensidad de emociones pasadas y presentes para un mismo contexto académico (actividad práctica).

- Analizar las asociaciones entre las emociones pasadas y el aprendizaje de Biología adquirido durante esta etapa.

\section{METODOLOGÍA}

\section{Participantes}

La muestra consiste en 152 futuros maestros (22 ańos media; $64 \%$ mujeres) pertenecientes a tres grupos de estudiantes que cursaban la asignatura Didáctica del Medio Físico y los Seres Vivos, del tercer curso del Grado en Educación Primaria de la Universidad de Extremadura. La muestra no es aleatoria, ya que se han elegido representantes de la población a los que se ha tenido acceso. Por tanto, los resultados no son extrapolables más allá del contexto local que representan: una muestra diversa de maestros en formación de Extremadura cuyo último contacto con las asignaturas de Biología fue, en la mayor parte de los casos, durante la Educación Secundaria (el 69 \% de los estudiantes no cursaron un Bachillerato de Ciencias), hace como mínimo 5 años. 


\section{Instrumento}

El instrumento utilizado (anexo I) es un cuestionario autoinforme con el que se determinan las emociones (primera parte) y se evalúan conceptos básicos de Biología de Educación Secundaria (segunda parte). En la primera parte se pide al alumno que autoinforme sobre 10 emociones académicas: 5 positivas (alegría, confianza, satisfacción, entusiasmo y diversión) y 5 negativas (preocupación, frustración, incertidumbre, nerviosismo y aburrimiento). Estas emociones han sido descritas previamente en muestras de maestros en formación inicial (Mellado et al., 2014) y tienen efecto sobre el aprendizaje (Pekrun, 2014). Para medir las emociones se emplean ítems simples, que comparados con test multiítem, proporcionan suficiente validez, requieren menos tiempo y son menos invasivos en las respuestas emocionales de los alumnos (Goetz et al., 2016). Los alumnos autoinforman, siguiendo una escala ordinal de Likert (que oscila desde 1 «no experimentada» a 5 «intensamente experimentada»), sobre la intensidad con la que habían experimentado o creían que iban a experimentar dichas emociones. El instrumento proporciona una medida simple, rápida y cuantitativa de las emociones, que apenas afecta al desarrollo de las actividades de aula, y se emplea para: i) determinar emociones pasadas (recuerdo de las emociones experimentadas por la muestra en su etapa como estudiantes de Educación Secundaria), durante las clases magistrales (desarrolladas bajo un enfoque expositivo tradicional) y durante las clases prácticas (desarrolladas bajo un enfoque experimental, entre las que se incluyen prácticas de laboratorio, salidas al medio natural y realización de ejercicios o trabajos grupales), y ii) determinar sus emociones presentes ante la expectativa de realizar una práctica de laboratorio en un seminario de la asignatura Didáctica del Medio Físico y los Seres Vivos.

Los conceptos básicos de Biología se evalúan a través de un cuestionario (15 preguntas de tipo test de Biología Celular) validado siguiendo los pasos indicados por Smith et al. (2008): i) revisión sobre concepciones alternativas relacionadas con la estructura y fisiología de la célula para la etapa inicial de Educación Secundaria (Marcos-Merino y Esteban, 2018); ii) en base a esos contenidos, selección de objetivos de aprendizaje propios del segundo curso de la Educación Secundaria Obligatoria (ESO), nivel en ciencias más alto alcanzado por la mayoría de la muestra, y iii) elaboración de preguntas propias y selección de preguntas del TIMSS (Trends in International Mathematics and Science Study) (Foy, Arora y Stanco, 2013). De esta manera el cuestionario queda estructurado en tres secciones: S1, preconcepciones; S2, conceptos básicos; y S3, preguntas del TIMSS. Así, este cuestionario examina diferentes niveles de comprensión de la Biología Celular (célula, estructura y función, incluyendo el material hereditario). Las puntuaciones de cada una de las secciones se trataron como variables centradas y reducidas $(0 \pm 1$ media $\pm \mathrm{SD})$. El conocimiento de Biología se estimó a partir de la media de estos tres valores centrados.

\section{Procedimiento}

Los estudiantes cumplimentaron los cuestionarios antes de comenzar un seminario práctico de la asignatura Didáctica del Medio Físico y los Seres Vivos (extracción de ADN con materiales cotidianos) en el que se abordaron distintos contenidos de Biología Celular y su didáctica (Esteban Gallego, MarcosMerino, Gómez Ochoa de Alda, 2019. La realización de esta práctica ya ha revelado una asociación significativa entre las emociones y el aprendizaje adquirido durante esta (Marcos-Merino, Esteban y Ochoa de Alda, 2016) con una muestra de alumnos similar. Los alumnos informaron primero sobre sus emociones para evitar que el examen de conocimientos interfiriese en sus emociones. Los estudiantes fueron informados del carácter voluntario y anónimo de su participación. El tiempo medio empleado para cumplimentar el cuestionario osciló entre 10 y 15 minutos. 


\section{Análisis estadístico}

Dado que los datos no se ajustaban a una distribución normal se utilizó estadística no paramétrica. Los datos de los tres grupos de estudiantes se agruparon en un único conjunto de datos, tras comprobar la ausencia de diferencias significativas entre ellos. Los test de normalidad (Kolmogoro-Smirnov y Shapiro-Wilk), el análisis de correlación (Spearman) y el análisis factorial se realizaron con el programa SPSS. Para la extracción de los factores se emplearon mínimos cuadrados generalizados y una rotación Oblimin. Este método de extracción es robusto frente a la carencia de normalidad de los datos y proporciona el test $\chi^{2}$ con el que evaluar el modelo. El modelo factorial se considera factible cuando los datos observados en el estudio y los esperados con el modelo no muestran diferencias significativas (test- $\chi^{2}$, p-valor $\left.>0,05\right)$. Una vez calculados, los factores fueron guardados como variables centradas. En todos los casos la fiabilidad (alfa de Cronbach) y la medida de adecuación muestral (Kaiser-MeyerOlkin) eran mayores que 0,7 y 0,8 respectivamente. Las pruebas no paramétricas empleadas para comparar grupos (test de Wilcoxon), las gráficas y las regresiones lineales se realizaron con el programa KaleidaGraph. Los datos obtenidos en el estudio están a disposición de todo aquel que lo solicite.

\section{RESULTADOS Y DISCUSIÓN}

\section{Los alumnos recuerdan sus emociones con diferente intensidad}

Salvo en el caso de la preocupación y la incertidumbre, los estudiantes del Grado en Educación Primaria describen con una intensidad significativamente diferente cada una de las emociones que experimentaron durante las clases expositivas y prácticas de ciencias de Educación Secundaria ( $\mathrm{p}$-valor < 0,05 , test de Wilcoxon) (figura 1). El recuerdo de las emociones positivas es, en todos los casos, más intenso en clases prácticas que en las expositivas. En los casos de las emociones alegría, entusiasmo y diversión, la mediana aumenta de 2 en clases expositivas a 4 en prácticas ( $\mathrm{p}$-valor $<0,001$ ); mientras que la mediana de la satisfacción aumenta de 3 en clases magistrales a 4 en prácticas ( $\mathrm{p}$-valor $<0,001$ ). Respecto a la confianza (mediana de 3 para ambos contextos), las diferencias significativas observadas ( $\mathrm{p}$-valor $<0,001)$ se deben a un aumento de la varianza hacia valores superiores. En relación con el recuerdo de emociones negativas, la mayoría son más intensas en clases teóricas. Este es el caso del aburrimiento y la preocupación (la mediana de ambas es de 3 en clases expositivas y 2 en prácticas, p-valor $<0,001$ ); así como de la frustración (mediana de 2 para ambos contextos). Las diferencias significativas observadas para esta emoción ( $\mathrm{p}$-valor $<0,001$ ) se deben a una disminución de la varianza. La única emoción negativa que los estudiantes recuerdan haber experimentado más en clases prácticas es el nerviosismo (mediana de 3 en clases prácticas y de 2 en expositivas, p-valor $<0,001$ ), mientras que no se observan diferencias significativas para la incertidumbre.

Los participantes en este estudio atribuyen una intensidad de emociones distinta para un mismo contexto académico pasado (clases prácticas como estudiantes de Educación Secundaria) y presente (clase práctica como maestros en formación). Concretamente, diferencian el recuerdo de la intensidad de alegría, diversión, nerviosismo ( $\mathrm{p}$-valor $<0,01$, test de Wilcoxon) y preocupación ( $\mathrm{p}$-valor $<0,001$, test de Wilcoxon). Este resultado sugiere, a diferencia de lo señalado por Robinson y Clore (2002), que los alumnos son capaces de recordar emociones pasadas y diferenciarlas de las emociones presentes hacia una situación determinada similar, desvinculado las emociones presentes de las pasadas. 


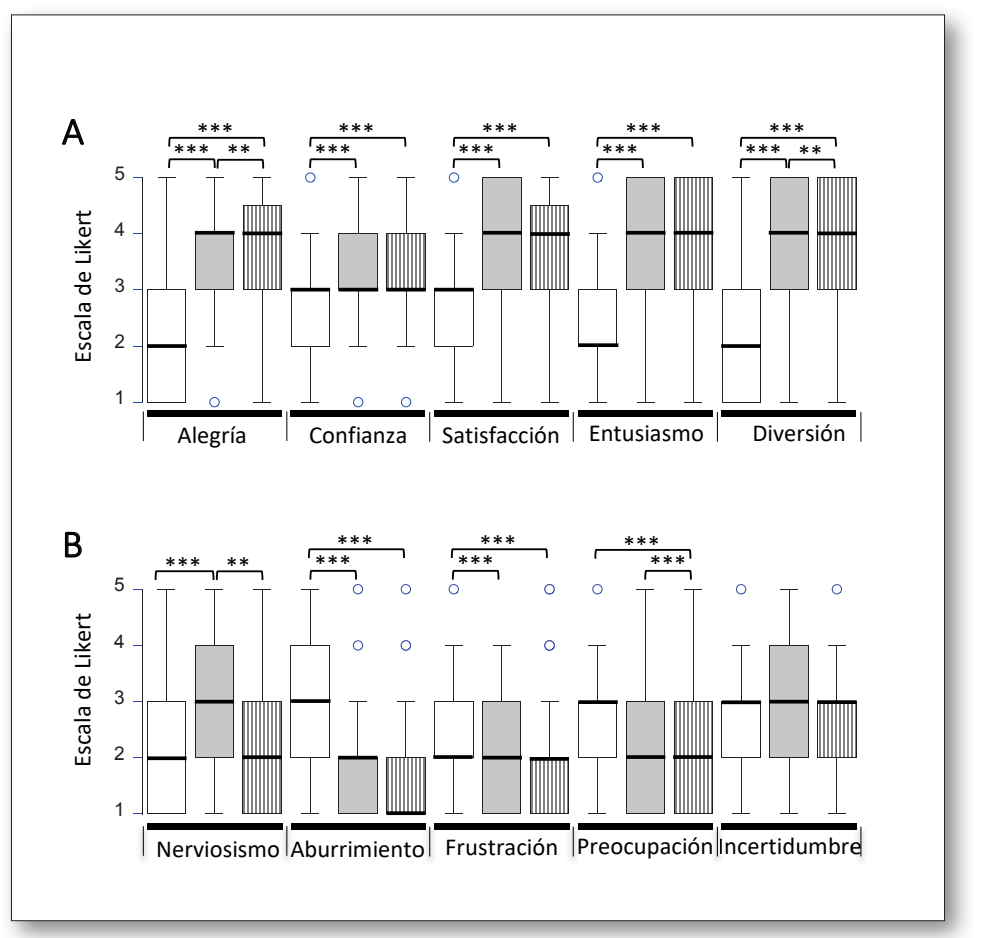

Fig. 1. Distribución de la intensidad de las emociones, positivas (A) y negativas (B), experimentadas de manera habitual por los participantes durante clases expositivas (cajas vacías), clases prácticas (cajas grises) y justo antes de realizar una práctica de extracción de $\mathrm{ADN}$ (cajas rayadas). La recta horizontal dentro de cada caja representa la mediana. Los límites inferiores y superiores de cada caja corresponden a los percentiles 25 y 75, y la terminación inferior y superior de las líneas verticales a los percentiles 5 y 95. Las líneas horizontales sobre las cajas unen grupos significativamente diferentes (test de Wilcoxon, ${ }^{* * *}$-valor $<0,001$, ** p-valor $<0,01)$.

\section{Validación del test autoinforme de emociones: matriz de intercorrelaciones y análisis factorial}

El análisis de las correlaciones entre la intensidad de las diferentes emociones muestra una asociación positiva entre aquellas correspondientes a una misma valencia (positiva o negativa) en cada una de las 3 situaciones analizadas: clases expositivas pasadas, prácticas pasadas y previas a la práctica del presente (tabla 1). Esto sugiere la presencia de factores latentes comunes a cada grupo de emociones, positivas y negativas, en estos tres contextos estudiados. Por otra parte, las emociones negativas no se correlacionan significativamente con las positivas. Esta falta de correlación, ya descrita previamente (Trigwell, Ellis y Han, 2012; Pekrun et al., 2011), indica que, en general, las emociones positivas tienen poco efecto modulador sobre las negativas y viceversa. Una excepción notable es el aburrimiento, emoción negativa que se correlaciona significativa y negativamente con todas las positivas. Esta circunstancia se observa también, aunque en menor medida, con la frustración.

El análisis factorial (tabla 2) confirma la existencia de factores latentes. Como se muestra en la matriz de estructura, que recoge la correlación de cada emoción con los dos factores extraídos para cada contexto académico (clases expositivas y prácticas pasadas, y antes de la práctica del presente), las emociones experimentadas en cada situación académica se pueden descomponer en dos factores. Estos corresponden a las dos valencias de las emociones (positivas o negativas). Así, el factor 1 correlaciona positivamente con las emociones positivas, mientras que el factor 2 lo hace con las negativas. En todos 
los casos el coeficiente alfa de Cronbach es superior a 0,7, el test KMO mayor a 0,8, el porcentaje de varianza explicada superior al $50 \%$ y la prueba de bondad del ajuste $\chi^{2}$ muestra que los datos proporcionados por el modelo factorial no son significativamente diferentes a los observados ( $\mathrm{p}$-valor $>0,05$ ). Todo ello sugiere que el modelo es plausible.

Tabla 1.

Matriz de intercorrelaciones (correlación de Spearman; ${ }^{* *}$ p-valor $<0,001$, ${ }^{*}$ p-valor $\left.<0,05\right)$ entre las intensidades de las emociones académicas analizadas previas a la práctica a realizar (normal), prácticas (subrayado) y clases expositivas pasadas (cursiva).

En negrita se resaltan las correlaciones significativas entre las emociones de una misma valencia.

\begin{tabular}{|c|c|c|c|c|c|c|c|c|c|c|}
\hline Emoción & (1) & (2) & (3) & (4) & (5) & (6) & (7) & (8) & (9) & (10) \\
\hline $\begin{array}{l}\text { Alegría } \\
\text { (1) }\end{array}$ & $\begin{array}{l}1 \\
1 \\
1\end{array}$ & $\begin{array}{l}.501^{* *} \\
.484^{* *} \\
.562^{* *}\end{array}$ & $\begin{array}{l}.728^{* *} \\
.619^{* *} \\
.531^{* *}\end{array}$ & $\begin{array}{l}.691^{* *} \\
.622^{* *} \\
.559^{* *}\end{array}$ & $\begin{array}{l}.702^{* *} \\
.608^{* *} \\
.565^{* *}\end{array}$ & $\begin{array}{l}.128 \\
.113 \\
.157\end{array}$ & $\begin{array}{l}-.277^{* *} \\
-.231^{* *} \\
-.328^{* *}\end{array}$ & $\begin{array}{r}-.101 \\
-.075 \\
-.105\end{array}$ & $\begin{array}{r}-.011 \\
-.004 \\
.059\end{array}$ & $\begin{array}{r}.142 \\
.104 \\
.048\end{array}$ \\
\hline $\begin{array}{c}\text { Confianza } \\
\text { (2) }\end{array}$ & & $\begin{array}{l}\frac{1}{1} \\
1\end{array}$ & $\begin{array}{l}.581^{* *} \\
.552^{* *} \\
.594^{* *}\end{array}$ & $\begin{array}{l}.393^{* *} \\
._{446^{* *}}^{.495^{* *}}\end{array}$ & $\begin{array}{l}.427^{* *} \\
.335^{* *} \\
.398^{* *}\end{array}$ & $\begin{array}{c}-.080 \\
.085 \\
.087\end{array}$ & $\begin{array}{l}-.207^{*} \\
-.126 \\
-.319^{* *}\end{array}$ & $\begin{array}{c}-.092 \\
.028 \\
-.200^{*}\end{array}$ & $\begin{array}{l}-.117 \\
.027 \\
-.063\end{array}$ & $\begin{array}{r}-.152 \\
.093 \\
-.032\end{array}$ \\
\hline $\begin{array}{c}\text { Satisfacción } \\
\text { (3) }\end{array}$ & & & $\begin{array}{l}1 \\
1 \\
1\end{array}$ & $\begin{array}{l}.705^{* *} \\
.679^{* *} \\
.607^{* *}\end{array}$ & $\begin{array}{l}.714^{* *} \\
.590^{* *} \\
.561^{* *}\end{array}$ & $\begin{array}{r}-.050 \\
-.031 \\
.169^{*}\end{array}$ & $\begin{array}{l}-.313^{* *} \\
-.327^{* *} \\
-.442^{* *}\end{array}$ & $\begin{array}{l}-.187^{*} \\
\frac{-.188^{*}}{-.202^{* *}}\end{array}$ & $\begin{array}{r}-.139 \\
-.118 \\
-.020\end{array}$ & $\begin{array}{l}-.009 \\
.118 \\
.072\end{array}$ \\
\hline $\begin{array}{c}\text { Entusiasmo } \\
(4)\end{array}$ & & & & $\begin{array}{l}1 \\
1 \\
1\end{array}$ & $\begin{array}{l}.722^{* *} \\
.614^{* *} \\
.731^{* *}\end{array}$ & $\begin{array}{r}-.057 \\
-.021 \\
.098\end{array}$ & $\begin{array}{l}-.435^{* *} \\
-.361^{* *} \\
-.414^{* *}\end{array}$ & $\begin{array}{l}-.284^{* *} \\
\frac{-.198^{*}}{-.206}\end{array}$ & $\begin{array}{r}-.152 \\
-.078 \\
-.052\end{array}$ & $\begin{array}{l}.042 \\
.134 \\
.071\end{array}$ \\
\hline $\begin{array}{l}\text { Diversión } \\
(5)\end{array}$ & & & & & $\begin{array}{l}1 \\
1 \\
1\end{array}$ & $\begin{array}{c}-.004 \\
.119 \\
.192^{*}\end{array}$ & $\begin{array}{l}-.312^{* *} \\
-.239^{* *} \\
-.378^{* *}\end{array}$ & $\begin{array}{r}-.160^{*} \\
-.003 \\
-.119\end{array}$ & $\begin{array}{r}-.030 \\
-.014 \\
.011\end{array}$ & $\begin{array}{r}.030 \\
-.136 \\
.078\end{array}$ \\
\hline Nerviosismo (6) & & & & & & $\begin{array}{l}1 \\
1 \\
1\end{array}$ & $\begin{array}{l}. \mathbf{4 6 1} \\
.267^{* *} \\
.070\end{array}$ & $\begin{array}{l}.593^{* *} \\
.552^{* *} \\
.183^{*}\end{array}$ & $\begin{array}{l}.561^{* *} \\
.550^{* *} \\
.393^{* *}\end{array}$ & $\begin{array}{l}.326^{* *} \\
.253^{* *} \\
.421^{* *}\end{array}$ \\
\hline $\begin{array}{c}\text { Aburrimiento } \\
\text { (7) }\end{array}$ & & & & & & & $\begin{array}{l}1 \\
1 \\
1\end{array}$ & $\begin{array}{l}.627^{* *} \\
.487^{* *} \\
.403^{* *}\end{array}$ & $\begin{array}{l}.566^{* *} \\
.^{* 400^{* *}} \\
.100\end{array}$ & $\begin{array}{l}.319^{* *} \\
.140 \\
.023\end{array}$ \\
\hline $\begin{array}{c}\text { Frustración } \\
\text { (8) }\end{array}$ & & & & & & & & $\begin{array}{l}1 \\
1 \\
1\end{array}$ & $\begin{array}{l}.730^{* *} \\
.682^{* *} \\
.490^{* *}\end{array}$ & $\begin{array}{l}.336^{* *} \\
.374^{* *} \\
.309^{* *}\end{array}$ \\
\hline $\begin{array}{c}\text { Preocupación } \\
\text { (9) }\end{array}$ & & & & & & & & & $\begin{array}{l}1 \\
1 \\
1\end{array}$ & $\begin{array}{l}.474^{* *} \\
.435^{* *} \\
.411^{* *}\end{array}$ \\
\hline $\begin{array}{l}\text { Incertidumbre } \\
\qquad(10)\end{array}$ & & & & & & & & & & $\begin{array}{l}1 \\
1 \\
1\end{array}$ \\
\hline
\end{tabular}


Tabla 2 .

Matriz de estructura obtenida tras el análisis factorial exploratorio realizado con los datos de intensidad de las emociones académicas en los tres contextos estudiados. Se resaltan en cursiva las correlaciones más elevadas $(\geq 0,250)$.

\begin{tabular}{|l|c|c|c|c|c|c|}
\hline & \multicolumn{2}{|c|}{ Clases expositivas pasadas } & \multicolumn{2}{c|}{ Clases prácticas pasadas } & \multicolumn{2}{c|}{$\begin{array}{c}\text { Previo a práctica } \\
\text { de extracción de ADN }\end{array}$} \\
\hline Emociones & Factor 1 & Factor 2 & Factor 1 & Factor 2 & Factor 1 & Factor 2 \\
\hline Alegría & .752 & -.139 & .729 & .085 & .841 & .020 \\
\hline Confianza & .620 & -.213 & .572 & .071 & .532 & -.294 \\
\hline Satisfacción & .772 & -.089 & .832 & -.126 & .835 & -.152 \\
\hline Entusiasmo & .891 & -.064 & .820 & .002 & .872 & -.094 \\
\hline Diversión & .720 & .028 & .720 & .084 & .796 & .016 \\
\hline Nerviosismo & -.017 & .602 & .117 & .693 & .057 & .680 \\
\hline Aburrimiento & -.505 & .296 & -.327 & .389 & -.301 & .617 \\
\hline Frustración & -.330 & .685 & -.125 & .832 & -.190 & .830 \\
\hline Preocupación & -.111 & .808 & .036 & .768 & -.040 & .864 \\
\hline Incertidumbre & -.095 & .689 & .115 & .409 & -.025 & .516 \\
\hline
\end{tabular}

Estos resultados sugirieren que el factor 1 corresponde a las emociones positivas mientras que el factor 2 corresponde a las negativas. Tanto la intercorrelación entre emociones de la misma valencia, como su ajuste a un modelo factorial, han sido observados ya en emociones estimadas mediante instrumentos más complejos que utilizan varios ítems para determinar cada emoción (Goetz et al., 2007; Pekrun et al., 2011).

En consonancia con lo observado en la matriz de intercorrelaciones, el aburrimiento muestra un comportamiento diferente ya que, además de correlacionarse positivamente con el factor 2 , se correlaciona negativamente con el factor 1 . Estos resultados son similares a los recogidos en las investigaciones de Pekrun et al. (2010), Pekrun et al. (2014) y de Trigwell et al. (2012), e indican que una intervención sobre esta emoción permite no solo atenuar las emociones negativas sino incrementar las positivas.

En conjunto, el análisis factorial y la concordancia de los resultados con estudios previos sugieren que el cuestionario autoinforme desarrollado para medir emociones, a pesar de su sencillez, permite capturar la valencia y la intensidad de las emociones presentes y pasadas experimentadas por un determinado grupo de alumnos. El hecho de que no se aprecie un único factor para las emociones positivas y otro para las negativas común a todos los contextos (sino uno para cada contexto) sugiere que las emociones experimentadas en un contexto determinado tienen poco efecto sobre el otro (p. ej. aumentar la intensidad de las emociones positivas en el laboratorio no tendría efecto sobre esas emociones en el aula).

\section{Análisis de las relaciones entre el recuerdo de emociones como estudiantes de Educación Secundaria y el aprendizaje duradero de Biología}

Los participantes en esta investigación muestran una gran heterogeneidad en relación con su grado de conocimiento de los conceptos básicos de Biología examinados en las secciones del cuestionario S1, S2 y S3. Los resultados obtenidos por los alumnos en cada una de las secciones no están significativamente correlacionados, lo que indica que evalúan aspectos diferentes del aprendizaje de la Biología Celular. Los conocimientos examinados en estos cuestionarios son el resultado de un aprendizaje de- 
sarrollado hace más de 5 años, ya que la formación previa de los participantes referente a aspectos de Biología Celular alcanzó como máximo, en la mayor parte de los casos, la Educación Secundaria. Se trata, por tanto, de un aprendizaje consolidado y duradero. Los estudios de correlación muestran una asociación significativa entre la intensidad de algunas emociones y la nota obtenida en las secciones $S 1$, S2 y S3 (figura 2). Los estudiantes que mostraron un mayor grado de recuerdo de conceptos básicos de Biología (S2) son los que recordaron haber experimentado con menor intensidad incertidumbre (Spearman -0,200, p-valor $<0,01$ ), preocupación (Spearman -0,190, p-valor $<0,01$ ) y nerviosismo (Spearman -0,160, p-valor $<0,01$ ) durante las clases expositivas; y los que obtuvieron mayor nota en las preguntas TIMSS (S3) recordaron haber experimentado menos nerviosismo (Spearman -0,243, p-valor $<0,001$ ) y frustración (Spearman $-0,159$, p-valor $<0,01$ ) en clases expositivas y menos preocupación (Spearman -0,192, p-valor $<0,01$ ), frustración (Spearman -0,174, p-valor $<0,01$ ) y aburrimiento (Spearman -0,253, p-valor < 0,001) en las clases prácticas de Educación Secundaria.

Respecto a las emociones positivas, los estudiantes que describieron más satisfacción, alegría y entusiasmo, durante las clases prácticas pasadas, eran los que obtenían mejores resultados en las secciones S1 y S3. Así, los resultados obtenidos en S1 se correlacionan con la satisfacción (Spearman 0,246, p-valor $<0,001$ ) y la alegría (Spearman 0,162, p-valor $<0,01$ ); mientras que los obtenidos en S3 se correlacionan con la satisfacción (Spearman 0,205, p-valor $<0,01$ ), la alegría (Spearman 0,161, p-valor < 0,01 ) y el entusiasmo (Spearman 0,182, p-valor $<0,01$ ). El entusiasmo y la diversión experimentados durante las clases expositivas presentan un comportamiento singular, ya que se asocian negativamente con la retención de los contenidos S2 (Spearman 0,161 con el entusiasmo, p-valor < 0,01) y S3 (Spearman 0,202 con la diversión, p-valor $<0,01)$. Este comportamiento concuerda con estudios previos que muestran cómo las emociones no manifiestan necesariamente un mismo efecto univalente sobre el aprendizaje (Tyng et al., 2017), y sugiere que tanto el entusiasmo como la diversión en las clases teóricas pueden tener un efecto más distractor que motivador sobre el aprendizaje de conceptos básicos. El análisis de los factores latentes mostró que los futuros maestros que obtuvieron mejores resultados en los conocimientos de Biología (promediado de S1, S2 y S3) describieron más emociones positivas durante las clases prácticas (Spearman 0,204, p-valor $<0,01$ ) y menos emociones negativas durante las clases expositivas (Spearman 0,206, p-valor $<0,001$ ).

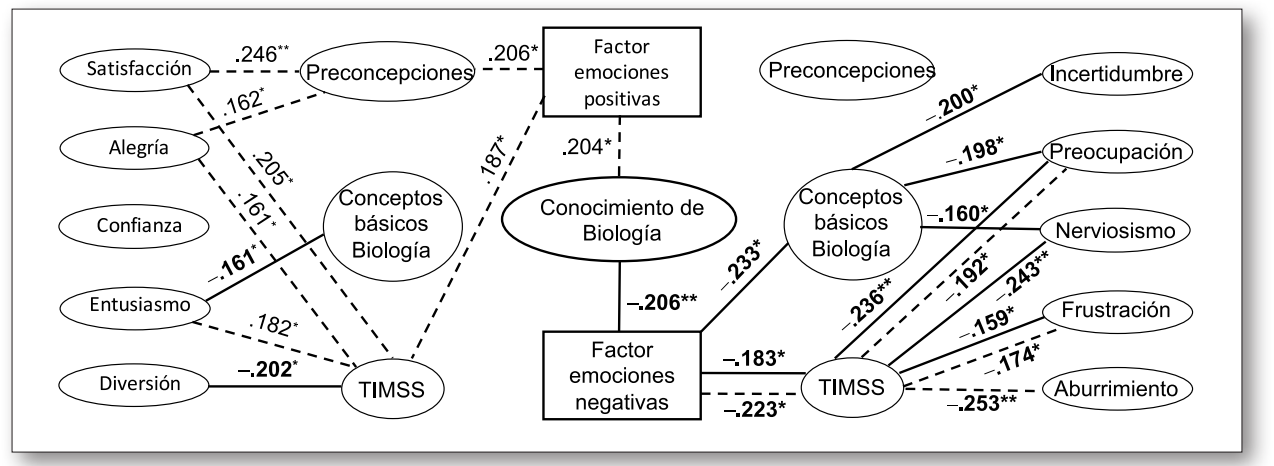

Fig. 2. Modelo que representa las correlaciones significativas (Spearman, ${ }^{* *}$ p-valor $<0,01$, ${ }^{*}$ p-valor $<0,05)$ entre los resultados obtenidos en las pruebas de conocimiento relativas a preconcepciones (S1), conocimientos básicos de Biología (S2) y preguntas del TIMSS (S3), y la intensidad de las emociones experimentadas durante las clases expositivas (líneas continuas) y prácticas (líneas discontinuas). En la parte central se muestran las correlaciones entre la estimación conjunta del conocimiento de Biología y el factor correspondiente a cada grupo de emociones (positivas y negativas) en cada contexto académico. Las correlaciones negativas se muestran en negrita. 
Estos resultados concuerdan con otros descritos previamente (Pekrun, 2006), según los cuales los resultados de aprendizaje de Matemáticas, Física e Inglés se correlacionan, en el corto plazo, positivamente con las emociones positivas y negativamente con las negativas, siendo diferentes para los distintos contextos académicos. Entre estas correlaciones destacan las correlaciones negativas entre los resultados de aprendizaje y el nerviosismo (Daniels et al., 2009; Pekrun, Elliot y Maier, 2009) y el aburrimiento (Daniels et al., 2009; Pekrun et al., 2009; Pekrun et al., 2014), correlaciones detectadas en la presente investigación para clases expositivas y prácticas, respectivamente.

Investigaciones recientes han constatado que existen relaciones bidireccionales entre los dominios cognitivo y afectivo (Eldar y Niv, 2015; Pekrun et al., 2017). Desde la perspectiva de la teoría del Control-Valor (Pekrun, 2006), se ha comprobado que las emociones académicas están significativamente relacionadas con logros académicos, pero existen pocos estudios sobre el mantenimiento de esta asociación a largo plazo. Los resultados de la presente investigación ponen de manifiesto que existe una asociación entre algunos aprendizajes duraderos de Biología, perpetuados desde la Educación Secundaria, y las emociones experimentadas por una muestra de maestros en formación, de la Universidad de Extremadura, en su etapa de estudiantes de Secundaria. La correlación positiva observada, entre los resultados de aprendizaje y el factor correspondiente al recuerdo de emociones positivas en las prácticas, concuerda con las aportaciones de Kensinger y Corkin (2004), quienes comprobaron que los estímulos emocionales positivos provocan, a nivel de la amígdala, una excitación que mejora la memoria. En su conjunto, los resultados obtenidos coinciden con lo señalado por distintas investigaciones previas, que han demostrado que la información relacionada con algún estímulo emocional se recuerda mejor que la información neutra (Dunsmoor et al., 2015), y con investigaciones que han puesto de manifiesto el papel que juegan las emociones en la modulación de la memoria (Gray, Braver y Raichle, 2002) y en su codificación y recuperación a largo plazo (Tyng et al., 2017). Sin embargo, la mayoría de estos trabajos se han realizado en relación con la memoria de trabajo (memoria activa) o a corto plazo, por lo que la asociación entre emociones académicas y memoria a largo plazo ha sido aún poco explorada.

\section{CONCLUSIONES}

Los instrumentos desarrollados nos han permitido determinar el recuerdo de la intensidad de diez emociones pasadas, experimentadas durante las clases expositivas y prácticas de ciencias de la ESO, en una muestra de maestros en formación. Estos recuerdan haber experimentado con más intensidad, en las clases prácticas, todas las emociones positivas analizadas (alegría, confianza, diversión, satisfacción y entusiasmo), y con menor intensidad las emociones negativas aburrimiento y frustración, lo que evidencia el papel modulador del contexto académico en las emociones. El análisis de las emociones presentes antes de una práctica muestra la misma diferencia en intensidades, lo que sugiere que ese papel modulador se mantiene a lo largo del tiempo.

Para un contexto académico similar, presente y pasado (prácticas de ciencias de ESO y universidad, respectivamente), las intensidades de la alegría, diversión, nerviosismo y preocupación son distintas y se agrupan en factores diferentes. Esto pone de manifiesto que la muestra de alumnos es capaz de autoinformar y discernir entre emociones presentes y pasadas en contextos educativos similares.

Los instrumentos utilizados nos han permitido analizar tanto la asociación entre el conocimiento de Biología Celular de los alumnos y sus emociones individuales, como la asociación entre ese conocimiento y los factores latentes correspondientes a las emociones positivas y negativas en cada contexto de aprendizaje. Los resultados evidencian una asociación positiva entre el factor correspondiente a las emociones positivas en las clases prácticas de la ESO y el nivel de conocimientos adquirido en esa etapa; así como una asociación negativa entre estos y el factor asociado a las emociones negativas en las 
clases expositivas de la ESO. De manera individual la incertidumbre, la preocupación y el nerviosismo podrían tener un efecto adverso en la adquisición de conceptos básicos en las clases teóricas, mientras que la preocupación, el nerviosismo, la frustración y el aburrimiento podrían tener un efecto adverso en el aprendizaje de la utilización de esos conocimientos en las clases teóricas y prácticas. Por el contrario, la satisfacción, la alegría y el entusiasmo experimentados en las prácticas podrían tener un efecto favorable sobre el aprendizaje, a la vez que el entusiasmo y la diversión experimentados en las clases teóricas tendrían un efecto adverso. En conjunto, los resultados muestran que existe una asociación duradera entre las emociones experimentadas en las clases de ciencias durante la Educación Secundaria y el aprendizaje adquirido en esa etapa. Esto sugiere que el valor subjetivo atribuido al aprendizaje de ciencias podría mantenerse a lo largo del tiempo y modular el aprendizaje permanente. Esto es particularmente importante en el caso de los maestros en formación, ya que una adecuada gestión de sus emociones no solo puede mejorar su formación, sino además puede promover su aprendizaje permanente y mejorar su futuro desempeño profesional.

\section{LIMITACIONES E IMPLICACIONES EDUCATIVAS}

La asociación duradera detectada entre las emociones y los resultados de aprendizaje de Biología de Secundaria no indica una determinada causalidad. No se puede inferir si los resultados de aprendizaje son consecuencia de una experiencia emocional positiva o negativa en el aula, o bien, si dichos resultados han determinado las emociones que recuerdan los estudiantes. Se requieren, por tanto, futuros estudios con los que conocer con más detalle el papel regulador de las emociones académicas en el aprendizaje. Independientemente, los resultados revelan que existe una asociación duradera entre ambas variables, acomodándose al papel de las emociones en el proceso adaptativo de los humanos: las personas están motivadas para recordar detalles de eventos emocionales, ya que esta información es útil para predecir y controlar acontecimientos importantes del futuro (Dunsmoor et al., 2015).

Otra limitación del presente estudio está relacionada con el número de participantes, ya que este es reducido y perteneciente a una población muy concreta: conocimientos de Biología en maestros en formación inicial. A pesar de esto, los resultados obtenidos concuerdan con estudios previos realizados con muestras más amplias y pertenecientes a otras poblaciones (Goetz et al., 2007; Trigwell et al., 2012). La mayor parte de estas investigaciones se han realizado en relación con materias asociadas a emociones negativas (Matemáticas, Física, Química o idiomas). El hecho de que obtengamos resultados congruentes investigando las emociones en el aprendizaje de la Biología, una disciplina asociada a emociones positivas, refuerza el interés del trabajo y amplía el papel de las emociones en el aprendizaje de las ciencias.

Aunque no se puede establecer una relación causa-efecto entre variables cognitivas y emocionales, los resultados de esta investigación, junto con otros ya mencionados, apoyan que una intervención educativa en la que se consideren las emociones podría mejorar el aprendizaje presente y futuro. La baja intercorrelación, o ausencia de ella, entre las emociones de distinta valencia y la ausencia de correlación entre los factores latentes correspondientes a las emociones positivas y negativas en cada contexto de aprendizaje indica que, en el aula, las emociones tienen que ser gestionadas de manera independiente. Salvo excepciones, no es previsible que una disminución de las emociones negativas tenga como consecuencia un aumento de las positivas. Tampoco es previsible que la modulación de las emociones en un contexto académico tenga efecto sobre las emociones en otro contexto. Los coeficientes de correlación, entre los factores de emociones positivas y negativas y el conocimiento de Biología, muestran en qué medida una modificación en las emociones experimentadas por el alumnado, en el aula o en el laboratorio, podría mejorar los resultados. Este trabajo de investigación sugiere que el 
aprendizaje podría ser favorecido si se disminuyesen de manera general las emociones negativas, tanto en clases expositivas como en prácticas, y se aumentasen las emociones positivas en estas últimas. En particular, se aconseja intervenir en primer lugar sobre el aburrimiento y la frustración ya que, atenuando ambas emociones negativas, se aumentan las positivas, lo que podría mejorar el aprendizaje tanto en las clases expositivas como en las prácticas. Otra aportación importante de este trabajo es el desarrollo de un sencillo cuestionario autoinforme cuantitativo con el que estimar, de manera rápida, la intensidad de ciertas emociones en el aula, lo que permitiría orientar sobre las emociones sobre las que hay que intervenir.

\section{AGRADECIMIENTOS}

Este estudio ha sido financiado por el Proyecto EDU2016-77007-R del Ministerio de Economía y Competitividad y por la Ayuda a Grupos GR1 8004 de la Junta de Extremadura y el Fondo de Desarrollo Regional. José María Marcos-Merino es beneficiario de una beca FPU del Ministerio de Educación, Cultura y Deportes.

\section{REFERENCIAS BIBLIOGRÁFICAS}

Damasio, A. (2010). Y el cerebro creó al hombre. Barcelona: Destino.

Daniels, L., Stupnisky, R., Pekrun, R., Haynes, T., Perry, R. y Newall, N. (2009). A longitudinal analysis of achievement goals: From affective antecedents to emotional effects and achievement outcomes. Journal of Educational Psychology, 101(4), 948-963. https://doi.org/10.1037/a0016096

Dunsmoor, J., Murty, V., Davachi, L. y Phelps, E. (2015). Emotional learning selectively and retroactively strengthens memories for related events. Nature, 520, 345-348. https://doi.org/10.1038/nature14106

EldAR, E. y Niv, Y. (2015). Interaction between emotional state and learning underlies mood instability. Nature communications, 6, 6149-6159.

https://doi.org/10.1038/ncomms7149

Eldar, E., Rutledge, R., Dolan, R. y Niv, Y. (2016). Mood as representation of momentum. Trends in Cognitive Sciences, 20(1), 15-24.

https://doi.org/10.1016/j.tics.2015.07.010

Esteban Gallego, R., Marcos-Merino, J. M., y Gómez Ochoa de Alda, J. A. (2019). Extracción de ADN con material cotidiano: diseño, implementación y validación de una intervención activa interdisciplinar. Educación Química, 30 (1), 42-57.

http://dx.doi.org/10.22201/fq.18708404e.2019.1.67658

Foy, P., Arora A. y Stanco, G. (2013). TIMSS 2011 User Guide for the International Database. Supplement 1: International Version of the TIMSS 2011 Background and Curriculum Questionnaires. Amsterdam: International Association for the Evaluation of Educational Achievement.

Frenzel, A., Goetz, T., Lüdtke, O., Pekrun, R. y Sutton, R. (2009). Emotional transmission in the classroom: exploring the relationship between teacher and student enjoyment. Journal of Educational Psychology, 101(3), 705-716. https://doi.org/10.1037/a0014695

Goetz, T., Frenzel, A., Pekrun, R., Hall, N. y Lüdtke, O. (2007). Between-and within-domain relations of students' academic emotions. Journal of Educational Psychology, 99(4), 715-733. https://doi.org/10.3389/fpsyg.2014.01153 
Goetz, T., Sticca, F., Pekrun, R., Murayama, K. y Elliot, A. (2016). Intraindividual relations between achievement goals and discrete achievement emotions: an experience sampling approach. Learning and Instruction, 41, 115-125. https://doi.org/10.1016/j.learninstruc.2015.10.007

Gray, J., Braver, T. y Raichle, M. (2002). Integration of emotion and cognition in the lateral prefrontal cortex. Proceedings of the National Academy of Sciences of the United States of America, 99(6), 4115-4120. https://doi.org/10.1073/pnas.062381899

Gu, X., Liu, X., VAn Dam, N., Hof, P. y Fan, J. (2013). Cognition-emotion integration in the anterior insular cortex. Cerebral Cortex, 23(1), 20-27. https://doi.org/10.1093/cercor/bhr367

Kassam, K., Markey, A., Cherkassky, V., Loewenstein, G. y Just, M. (2013). Identifying emotions on the basis of neural activation. PloS one, 8(6), 1-12. https://doi.org/10.1371/journal.pone.0066032

Kensinger, E. y Corkin, S. (2004). Two routes to emotional memory: Distinct neural processes for valence and arousal. Proceedings of the National Academy of Sciences of the United States of America, 101(9), 3310-3315.

https://doi.org/10.1073/pnas.0306408101

Marcos-Merino, J. M. y Esteban R. (2018). Concepciones alternativas sobre Biología Celular y Microbiología de los maestros en formación: implicaciones de su presencia. Campo Abierto, 6(2), 167-179.

Marcos-Merino, J. M., Esteban, R. y Ochoa de Alda, J. A. G. (2016). Efecto de una práctica docente diseñada partiendo de las emociones de maestros en formación bajo el enfoque Ciencia, Tecnología y Sociedad. Indagatio Didactica, 8(1), 143-157.

Mauss, I. y Robinson, M. (2009). Measures of emotion: A review. Cognition and emotion, 23(2), 209-237.

https://doi.org/10.1080/02699930802204677

Mega, C., Ronconi, L. y De Beni, R. (2014). What makes a good student? How emotions, selfregulated learning, and motivation contribute to academic achievement. Journal of Educational Psychology, 106(1), 121-131.

https://doi.org/10.1037/a0033546

Mellado, V., Borrachero, A., Dávila, M., Melo, L. y Brígido, M. (2014). Las emociones en la enseñanza de las ciencias. Enseñanza de las ciencias, 32, 11-36. https://doi.org/10.5565/rev/ensciencias. 1478

Murayama, K., Pekrun, R., Lichtenfeld, S. y Vom Hofe, R. (2013). Predicting long-term growth in students' mathematics achievement: The unique contributions of motivation and cognitive strategies. Child development, 84(4), 1475-1490. https://doi.org/10.1111/cdev.12036

Pekrun, R. (2006). The control-value theory of achievement emotions: Assumptions, corollaries, and implications for educational research and practice. Educational psychology review, 18(4), 315-341. https://doi.org/10.1007/s10648-006-9029-9

Pekrun, R. (2014). Emotions and learning. Ginebra: International Academy of Education/International Bureau of Education.

Pekrun, R., Elliot, A. y Maier, M. (2009). Achievement goals and achievement emotions: Testing a model of their joint relations with academic performance. Journal of Educational Psychology, 101(1), 115-135. b

https://doi.org/10.1037/a0013383 
Pekrun, R., Goetz, T., Daniels, L., Stupnisky, R. y Perry, R. (2010). Boredom in achievement settings: Exploring control-value antecedents and performance outcomes of a neglected emotion. Journal of Educational Psychology, 102(3), 531-549. https://doi.org/10.1037/a0019243

Pekrun, R., Goetz, T., Frenzel, A., Barchfeld, P. y Perry, R. (2011). Measuring emotions in students' learning and performance: The Achievement Emotions Questionnaire (AEQ). Contemporary educational psychology, 36(1), 36-48. https://doi.org/10.1016/j.cedpsych.2010.10.002

Pekrun, R., Hall, N., Goetz, T. y Perry, R. (2014). Boredom and academic achievement: Testing a model of reciprocal causation. Journal of Educational Psychology, 106(3), 696-710. https://doi.org/10.1037/a0036006

Pekrun, R., Lichtenfeld, S., Marsh, H. W., Murayama, K. y Goetz, T. (2017). Achievement emotions and academic performance: Longitudinal models of reciprocal effects. Child Development, 88(5), 1653-1670. https://doi.org/10.1111/cdev.12704

Putwain, D., Becker, S., Symes, W. y Pekrun, R. (2017). Reciprocal relations between students' academic enjoyment, boredom, and achievement over time. Learning and Instruction, in Press. https://doi.org/10.1016/j.learninstruc.2017.08.004

Robinson, M. y Clore, G. (2002). Belief and feeling: evidence for an accessibility model of emotional self-report. Psychological bulletin, 128(6), 934. https://doi.org/10.1037//0033-2909.128.6.934

Smith, M. Wood, W. y Knight, J. (2008). The genetics concept assessment: a new concept inventory for gauging student understanding of genetics. CBE-Life sciences Education, 7(4), 422-430. https://doi.org/10.1187/cbe.08-08-0045

Trigwell, K., Ellis, R. y Han, F. (2012). Relations between students' approaches to learning, experienced emotions and outcomes of learning. Studies in Higher Education, 37(7), 811-824. https://doi.org/10.1080/03075079.2010.549220

Tyng, C. M., Amin, H. U., SAAD, M. N. y Malik, A. S. (2017). The influences of emotion on learning and memory. Frontiers in Psychology, 8, 1454.

https://doi.org/10.3389/fpsyg.2017.01454 


\section{ANEXO I}

Esta actividad forma parte de un proyecto de investigación sobre la importancia de las emociones en el aula. En las futuras publicaciones los datos se mostrarán globalmente, preservando completamente el anonimato de los participantes. La participación en esta actividad es completamente voluntaria.

Sexo: V / H Modalidad de Bachillerato o FP realizada:

Parte 1. Señala las emociones que has experimentado en Educación Secundaria con la enseñanza de las ciencias basada en un enfoque expositivo y en un enfoque práctico y el grado con que las recuerdas, así como las emociones previas a la práctica de extracción de $\mathrm{ADN}$ a realizar en el seminario (de 1 -nada- a 5-mucho o intensamente-).

\begin{tabular}{|c|c|c|c|c|c|c|c|c|c|c|c|c|c|c|c|}
\hline & \multicolumn{5}{|c|}{$\begin{array}{l}\text { Clases expositivas de Edu- } \\
\text { cación Secundaria }\end{array}$} & \multicolumn{5}{|c|}{$\begin{array}{l}\text { Clases prácticas de Educa- } \\
\text { ción Secundaria }\end{array}$} & \multicolumn{5}{|c|}{$\begin{array}{l}\text { Expectativa ante la práctica } \\
\text { de extracción de } A D N\end{array}$} \\
\hline & 1 & 2 & 3 & 4 & 5 & 1 & 2 & 3 & 4 & 5 & 1 & 2 & 3 & 4 & 5 \\
\hline Alegría & & & & & & & & & & & & & & & \\
\hline Frustración & & & & & & & & & & & & & & & \\
\hline Satisfacción & & & & & & & & & & & & & & & \\
\hline Nerviosismo & & & & & & & & & & & & & & & \\
\hline Diversión & & & & & & & & & & & & & & & \\
\hline Incertidumbre & & & & & & & & & & & & & & & \\
\hline Entusiasmo & & & & & & & & & & & & & & & \\
\hline Preocupación & & & & & & & & & & & & & & & \\
\hline Confianza & & & & & & & & & & & & & & & \\
\hline Aburrimiento & & & & & & & & & & & & & & & \\
\hline
\end{tabular}

Parte 2. Responde a las siguientes preguntas (solo una opción correcta)

1. Podemos extraer material genético de:
a) Solo los seres vivos
b) Solo los procariotas
c) Solo los eucariotas
d) De todos los seres vivos y algunos no vivos (por ejemplo, virus)

2. ¿Dónde crees que se encuentra el ADN en un tomate?:
a) Solo en las células de la semilla
b) Solo en las células de la pulpa (del fruto)
c) Solo en las células de la piel
d) En todas las células

3. El ADN es:
a) Un ácido nucleico
b) Una grasa rodeada de fósforo
c) Una proteína
d) Un aminoácido 
4. En los humanos los cromosomas sexuales se encuentran exclusivamente en:
a) Las células de los testículos y los ovarios
b) Todas las células con núcleo
c) Los espermatozoides y los óvulos
d) Las mitocondrias de todas las células

5. Qué tipos de células tienen mitocondrias y cloroplastos:
a) Las células animales
b) Las células vegetales
c) Las bacterias
d) Ninguna, o bien tienen mitocondrias o bien tienen cloroplastos

6. La diferencia esencial entre célula procariota y célula eucariota radica en:
a) El tamaño celular
b) La pared celular
c) El núcleo celular
d) La composición química del citoplasma

7. Respecto al ADN señala la respuesta CORRECTA:
a) Se encuentra exclusivamente en el núcleo celular
b) Es el único ácido nucleico de la célula
c) Se encuentra en cloroplastos, mitocondrias y núcleo
d) Está formado por aminoácidos

\section{Señala la afirmación CORRECTA:}

a) Todas las células están rodeadas por una membrana plasmática rígida

b) La membrana plasmática está formada fundamentalmente por lípidos, proteínas, carbohidratos y nucleótidos

c) Dentro de las células algunos orgánulos están rodeados por membranas similares a la membrana plasmática

d) Solo las células eucariotas tienen membrana plasmática

9. En los vegetales, la pared celular es:
a) Una estructura extracelular formada por polisacáridos, fundamentalmente celulosa
b) Una estructura rígida que las rodeas
c) La materia prima para formar el papel
d) Todas las respuestas son correctas

10. Identifica la relación «tipo celular - características» INCORRECTA:
a) Todas las células vegetales tienen pared celular
b) Todas las células animales tienen mitocondrias y cloroplastos
c) Todas las células vegetales tienen mitocondrias y cloroplastos
d) Todas las células animales tienen mitocondrias

11. Los riñones son órganos que se encuentran en el cuerpo humano. A un hombre le sacaron uno de sus dos riñones cuando era joven porque estaba enfermo. Ahora tiene un hijo. ¿Cuántos riñones tuvo su hijo al nacer?
a) 1 , ya que solo se puede heredar lo que se tiene
b) 2, ya que no se han alterado sus genes
c) 2 , ya que los rińones se regeneran
d) 1 o 2 según si hereda 1 o ninguno del padre y el de la madre

12. ¿Cuál de las siguientes es la mejor descripción del propósito de la respiración celular?
a) Proporcionar energía para la actividad celular
b) Producir azúcar para almacenar en las células
c) Liberar oxígeno para la respiración
d) Proporcionar dióxido de carbono para la fotosíntesis 
13. ¿Qué tipo de células destruyen a las bacterias que invaden el cuerpo?
a) Los glóbulos blancos
b) Los glóbulos rojos
c) Las células del riñón
d) Las células del pulmón

14. La imagen muestra una célula. ¿Cuál es la función de la parte de la célula marcada con una X?
a) Almacenar agua
b) Producir alimento
c) Absorber energía
d) Controlar las actividades

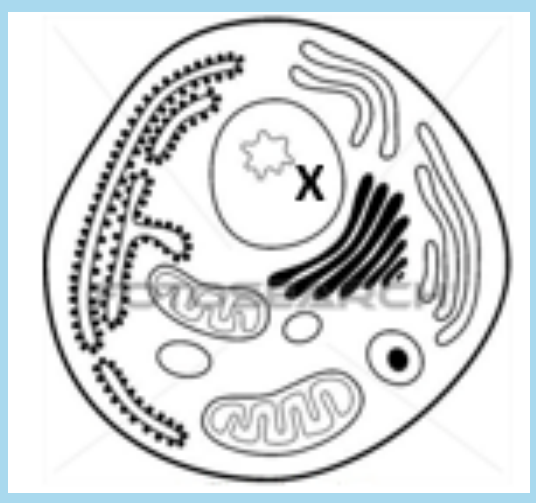

Con fondo verde se resaltan las preguntas sobre concepciones alternativas de Biología Celular

Con fondo gris se resaltan las preguntas sobre conceptos básicos de Biología Celular de la etapa de Educación Secundaria

Con fondo azul se resaltan las preguntas del TIMSS para 2. ${ }^{\circ}$ de ESO sobre Biología Celular 


\title{
Academic emotions and the learning of biology, a long-lasting association
}

\author{
Jesús A. G. Ochoa de Alda, José María Marcos-Merino \\ Departamento de Didáctica de las Ciencias Experimentales y las Matemáticas, Universidad de Extremadura \\ ochoadealda@unex.es,jmmarcos@unex.es \\ Francisco Javier Méndez Gómez \\ Departamento de Psicología y Antropología, Universidad de Extremadura, Cáceres, España \\ fjmendezgomez@gmail.com \\ Vicente Mellado Jiménez, M. Rocío Esteban Gallego \\ Departamento de Didáctica de las Ciencias Experimentales y las Matemáticas, Universidad de Extremadura \\ vmellado@unex.es, rocioesteban@unex.es
}

Emotions are human responses that arise from a condition that a stimulus has a positive or negative value in a particular context and for particular goals. Academic settings and learning achievements are one of these contexts and goals with which students can experience most human emotions. Emotional state and learning are reciprocally conditioned. Whereas the expectation of a given achievement modulates emotions before or during related activities, the subjective value of the attained achievement will modulate future emotions towards similar goals. If this reciprocity is maintained over time, it could be possible to detect a correlation between a worthwhile achievement such as consolidated knowledge (acquired a long time ago) and past emotions experienced during the acquisition of this knowledge. In this contribution, we hypothesized that Biology learning outcomes, perpetuated from Secondary Education, could maintain an association with academic emotions experienced at this stage. To assess this possibility, we sample 152 pre-service teachers, being their latest course of Biology during Secondary Education. In addition, we develop a simple and fast quantitative self-report test measuring ten academic emotions at two different academic settings (lectures and practices), together with another questionnaire to assess the knowledge of biology (misconceptions, basic knowledge, and TIMSS level, Grade 8). Finally, to evaluate the main study hypotheses, we adopted non-parametric statistics and factor analysis.

In agreement with previous findings, results evidence the modulating effect of academic settings over emotions. Lecture setting was associated with higher boredom and frustration as well as lower positive emotions (particularly enthusiasm and fun) compared to practices. Within negative emotions, nervousness was more experienced in practices. Regarding the relationship between emotions and the knowledge of Secondary Education Biology contents, Spearman's correlations detected significant relationships between the mark obtained in the assessment of knowledge and the self-reported intensity for every single emotion. Students who showed a better knowledge were those who experienced less nervousness, worry and uncertainty in lectures and less boredom, frustration and worry in practices. Moreover, these students experienced more joy, satisfaction and enthusiasm in practices and less enthusiasm and fun in lectures.

Intercorrelations show that positive and negative emotions correlate low, which support discriminant, construct validity. By contrast, significant correlations among positive and negative emotions suggest the presence of an underlying common factor. In agreement, according to a reliable factor analysis (Cronbach's alpha $>0,7$, and $\chi^{2}$ tests $>0,05$ ), emotions can be modelled into two factors: one for positive and one for negative emotions. Low intercorrelations between these factors suggest that positive and negative emotions should be independently managed in the classroom (i. e. an increase in positive emotions has a low effect on negative ones). Spearman's correlation analysis showed that the knowledge of biology was positively associated with the factor emerged from positive emotions experienced during practices, whereas this knowledge was inversely associated with the factor of negative emotions experienced during lectures.

Altogether, results support a long-lasting relation between emotions experienced during Biology courses in Secondary Education and the knowledge of biology consolidated from this stage. This suggests that the subjective value attributed to biology could be maintained long-term which, in turn, could modulate long-life learning, an important aspect for the future professional performance of pre-service teachers. 
